\title{
Induction of Apoptotic Death and Cell Cycle Arrest in HeLa Cells by Extracellular Factors of Breast Cancer Cells
}

\author{
Devashree Jahagirdar ${ }^{1}$, Charusheela R Gore ${ }^{2}$, Himadri Patel ${ }^{1}$, Kunjal Maria ${ }^{2}$, \\ Ishita Tandon', Nilesh K. Sharma ${ }^{1 *}$
}

\begin{abstract}
Background: There are evidences on the role of extracellular factors in cellular communication between cancer cells and non-cancerous cells to support tumor progression and a phenomenon of cancer cachexia. However, evidences are scarce to show the effects of extracellular factors from one carcinoma microenvironment upon growth and survival of another carcinoma. Methodology: To address the above issue, we have selected excised breast carcinoma tissue samples and in vitro grown MCF-7 sources of extracellular factors and tested their effects to evaluate growth and proliferation inhibitory potential against a cervical carcinoma cell line HeLa. Results: Data from the in vitro experiments like Trypan blue dye exclusion, MTT assay, cell cycle assay and annexin V/PI staining lead us to suggest that the extracellular factors collected from the culture medium of in vitro grown MCF-7 and excised breast carcinoma tissue play an apoptosis inducing and cell cycle arrest role in HeLa. In these in vitro experiments, we detected the presence of up to $40-50 \%$ apoptotic cell death in HeLa cells and increase in G2-M cell cycle phase from 11\%- $25 \%$ due to treatment with extracellular factors from human breast carcinoma cells. Discussion and Conclusion: These observations are novel and suggest that extracellular factors from breast carcinoma play an apoptosis inducing and growth inhibitory role upon on HeLa cells. This study can also support the concept of cancer cachexia and a possible hypothesis for rare chance of synchronous two or more primary tumor in a single patient.
\end{abstract}

Keywords: Heterogeneity- growth- death- neoplasms- microenvironment

Asian Pac J Cancer Prev, 19 (12), 3307-3316

\section{Introduction}

Tumor microenvironment provides an amiable niche which promotes the growth and progression of the carcinoma. Several reports in the literature suggest the role of tumor microenvironment in drug resistance and relapse of cancer (Marusyk et al., 2012; Meacham and Morrison, 2013; Holohan et al., 2013; Ahuja et al., 2016). A major cause behind cancer survival, progression, metastasis, and drug resistance that has been attributed is the microenvironmental heterogeneity of tumor (TMH) (Hanahan and Weinberg, 2011; Marusyk et al., 2012; Burrell et al., 2013; Meacham and Morrison, 2013; Chung et al. 2014; Alizadeh et al., 2015; Gkretsi et al., 2015; Yap et al., 2015; Sharma et al., 2016; Turner et al., 2017).

Importantly, tumor development and progression is supported by the non-cancerous tumor associated stromal and immune cells and extracellular factors which collectively are referred as TMH (Hanahan and Weinberg, 2011; Marusyk et al., 2012; Meacham and Morrison, 2013; Alizadeh et al., 2015; Yap et al., 2015; Sharma et al., 2016). The extracellular factors in particular have been indicated to contribute towards drug resistance and appearance of crucial cancer hallmarks (Hanahan and Weinberg, 2011; Marusyk et al., 2012; Meacham and Morrison, 2013; Alizadeh et al., 2015; Yap et al., 2015; Sharma et al., 2016). Commonly, non-cellular components of TME have been reported to include various types of molecules such as proteins, growth factors, cytokines, proteoglycans, glycoproteins, extracellular matrix (ECM) structural proteins, signalling mediators, BMP group of proteins, small regulatory RNAs, DNA and metabolites (Hanahan and Weinberg, 2011; Marusyk et al., 2012; Meacham and Morrison, 2013; Yap et al., 2015; Yuan et al., 2016). However, there is a dearth of knowledge on the crosstalk between extracellular factors released from one cancer type upon the growth and survival of another carcinoma in the same individual. Currently, there are evidences to support cancer cachexia in patients, which can be explained by the contribution of tumor secreted non-cellular factors upon the dysfunctioning of healthy tissues (Holohan et al., 2013; Kirr et al., 2014; Yap et 
al., 2015; Yuan et al., 2016; Ahuja et al., 2016; Sung and Weaver, 2017; Alves et al., 2017; Zhang et al., 2017, Steinbichler et al., 2017; Weidle et al., 2017). Besides the significance of cancer cachexia, rare cases of multiple cancers can be answered by indentifying the extracellular factors from a cancer and determining their ability to show modulation of growth and survival of another cancer type.

In the present investigation, our focus has been on the effect of extracellular factors from breast cancer microenvironment on the growth and survival of HeLa cancer cell in vitro.

\section{Materials and Methods}

\section{Materials}

Cell culture reagents were purchased from Invitrogen India Pvt. Ltd. and Himedia India Pvt. Ltd. HeLa and MCF-7 cell lines were procured from National Centre of Cell Science (NCCS), Pune. The clinical carcinoma tissue samples were obtained from the Department of Pathology at Dr. D. Y. Patil Medical College, Hospital and Research Centre, Pimpri, India. Sample collections were performed under proper ethical consent of patients, and routine biochemical and pathological examinations were conducted to confirm the breast carcinoma tumor.

\section{Cell line maintenance and Seeding}

HeLa cells were cultured and maintained in DMEM (Dulbecco's Modified Eagles Medium) (Himedia) with high glucose at $37^{\circ} \mathrm{C}$ and supplemented with $10 \%$ NBCS (New Born Calf Serum) (Himedia) and penicillin and streptomycin $100 \mu \mathrm{g} / \mathrm{ml}$. HeLa cells were regularly passaged after trypsinization by incubating with Trypsin/ EDTA (Himedia) and subsequently deactivated by the addition of culture media. Next, cells were plated or diluted by ensuring the routinely recommended dilution and plating density into culture flask and cell culture plate. The viability of cells was determined before plating the cells in the culture flask and plates by Trypan blue dye exclusion method.

\section{Histopathology and Immunohistochemistry of breast carcinoma samples}

An intra-operative breast lump biopsy from already diagnosed breast carcinoma patients was carried out under aseptic conditions. A portion of breast carcinoma tissue was also collected in DMEM complete medium and was used for the preparation of extracellular factors within $34 \mathrm{~h}$. For histopathology, these specimens were fixed in 10\% buffered formalin overnight, for an average period of $24 \mathrm{~h}$. Protocol for routine tissue processing was followed for histopathological examination (HPE). Second, the Peroxidase anti-peroxidase (PAP) method of immunohistochemistry was followed. Biogenex reagents were used for the antigen retrieval and immunohistochemistry IHC staining process. Biogenex Tris- EDTA based antigen retrieval solution with a $\mathrm{pH}$ of 9 and 6 was used for ER, PR staining and HER-2/ neu staining respectively. The heating cycles followed in the Biogenex temperature controlled microwave were two cycles of $10 \mathrm{~min}$ and $5 \mathrm{~min}$ each at $95^{\circ} \mathrm{C}$, with intermittent refilling of the antigen retrieval solution. Thereafter the slides were taken through the steps of wash with Tris buffer, peroxide block, power block and monoclonal antibodies. After this, slides were again washed in Tris buffer, the secondary antibody was applied and diamino benzidine chromogen was added. The slides were washed with water, and counterstained with hematoxylin. Then slides were serially dehydrated in alcohol, cleared in xylene and thereafter mounted using Distrene dibutyl phthalate xylene. After drying, the test slides were examined along with the control sections stained simultaneously.

Preparation of Extracellular factors of MCF-7 (EFM)

For the preparation ofextracellular factors of MCF-7 in DMEM medium, $0.7 \times 10^{6} \mathrm{MCF}-7$ cells were seeded according to the standard seeding protocol for T-75 flask with routinely used complete DMEM media as described above. Next, MCF-7 cells were allowed to grow for $48 \mathrm{~h}$ with continuous observations. At the end of $48 \mathrm{~h}$, overlaid complete DMEM medium from the culture flask was aspirated, collected and centrifugedat 1,500 rpm for 3 min. The supernatant was collected in fresh culture tube and labelled as extracellular factors of MCF-7 (EFM). This collected EFM in DMEM was again observed under microscope to ensure that EFM is free from the presence of MCF-7 cells or debris to suggest EFM potentially contained secreted extracellular factors. Next, for growth and viability assays, we used 1:1 ratio of EFM mixed with fresh DMEM high glucose (complete) media to test the effect of EFM upon growth and survival of HeLa cells.

\section{Breast Carcinoma tissue processing and preparation of non-cellular secreted factors (BCEF)}

In total, three large surgically removed breast cancer tissue samples were used in this experiment for the preparation of extracellular factors of tumour microenvironment. In brief, large biopsy samples were chopped into small sections under complete aseptic condition and then transferred to sterile pre-weighed centrifuge tube. Further, each tissue section was aseptically macerated using mortar and pestle in the presence of $4 \mathrm{ml}$ of complete DMEM media per $0.7 \mathrm{~g}$ of breast carcinoma tissue for 10-15 min. We processed at least three representative intra-tumoral sections from each large biopsy tissue samples. Next, homogenized breast carcinoma tissue samples was then transferred to sterile culture tube and spun at 8,000 rpm for $10 \mathrm{~min}$ at $4^{\circ} \mathrm{C}$ to pellet down cells, fats and debris to obtain clear supernatant. Further, supernatant was filtered using sterile micro syringe filter of 0.45 micron pore size. Subsequently, for all mentioned growth and viability experiment, filtered supernatant was designated as breast carcinoma extracellular factors (BCEF), which certainly represents the secreted extracellular factors and without any presence of cells and debris. This method is partially similar and adapted from to a similar kind protocol (Liu et al., 2016). Here, BCEF was used as the representative source of non-cellular factors of breast carcinoma and used to in the proportion of 1:1 ratio with fresh DMEM high glucose (complete) media and designated as BCEF: DMEM (1:1) to assess effects on growth and survival of HeLa cells. 
Characterization of EFM: DMEM (1:1) and BCEF: $\operatorname{DMEM}(1: 1)$ culture media

As mentioned in the above step, BCEF was collected as supernatant and breast carcinoma cells and debris were obtained as pellet. We performed cell count assay using haemocytometer by suspending the pelleted pellets in DMEM complete media to ensure that examine majority of breast carcinoma cells are pelleted down and only non-cellular secreted are in the supernatant, which is referred as BCEF. In brief, whole cell lysates of MCF-7 and breast carcinoma cells were prepared by using incubation and homogenization with RIPA buffer. Next, protein content of these cell lysates and EFM: DMEM $(1: 1)$ and BCEF: DMEM $(1: 1)$ was done using standard Lowry's Method. SDS-PAGE profiling of whole cell lysates from MCF-7 and breast carcinoma, EFM: DMEM $(1: 1)$ and BCEF: DMEM (1:1) was done using standard protocol. To exclude the possibilities of presence of lysed cellular proteins into EFM: DMEM (1:1) and BCEF: DMEM (1:1), these above proteins samples used for SDS-PAGE were analysed for the presence of cellular protein by performing routine immunoblot assay. For these, the primary antibodies that were used as anti-p53 mouse monoclonal antibody $(1: 2,000)(\mathrm{sc}-126)$ and anti-cytochrome $\mathrm{b}$ goat polyclonal antibody $(1: 2,000)$ (sc-9509). For the immunoblot detection, we used VisiGlo ${ }^{\text {TM }}$ HRP Chemiluminescent Substrates and images were acquired using BIO-RAD chemi-imager. The $\mathrm{pH}$ of DMEM control medium, EFM: DMEM (1:1) and BCEF: DMEM (1:1) was calculated using calibrated $\mathrm{pH}$ meter to measure the hydrogen ion activity in the solution indicating the acidity or alkalinity.

Trypan blue dye exclusion assay to determine anti-proliferative and anti-survival effects of EFM and $B C E F$

HeLa cells were grown up to $60-70 \%$ confluency and plated into a six well plate at the seeding density of $1.5 \times 10^{5}$ cells per well. The DMEM volume was kept at $2 \mathrm{ml}$ and was allowed to adhere for next 16-18 h. After $16 \mathrm{~h}$, cells were allowed to grow in the presence of following conditions: DMEM Control medium, EFM: DMEM (1:1) and BCEF: DMEM (1:1). Importantly, DMEM medium control was prepared to truly represent the control with reference to EFM and BCEF containing medium. The control DMEM media was prepared in 1:1 proportion with fresh $1 \mathrm{ml}$ complete DMEM media and $1 \mathrm{ml}$ of $48 \mathrm{~h}$ incubated dummy culture flask containing DMEM medium. Similarly, we also prepared and tested control DMEM media to truly represent normal control for BCEF: DMEM treatment media. Cells were incubated in the presence of $2 \mathrm{ml}$ of DMEM Control medium, EFM: $\operatorname{DMEM}(1: 1)$ and BCEF: DMEM $(1: 1)$ for $48 \mathrm{~h}$ at $37^{\circ} \mathrm{C}$ in $5 \% \mathrm{CO}_{2}$. After incubation for $48 \mathrm{~h}$, media were removed and centrifuged to collect the floating/dead cells. Next, cells were washed with PBS and treated with trypsin/ EDTA to harvest cells. Further, fresh media was added to inactivate trypsin and centrifuged for $3 \mathrm{~min}$ in sterile culture tube at $1500 \mathrm{rpm}$. Finally, cells were counted using Trypan blue dye exclusion assay to record live and dead cell on hemacytometer (Louis et al., 2011; Strober, 2015).

\section{MTT based cytotoxicity assay}

HeLa cells were seeded into 96 well flat bottom plate with the seeding density of 10,000 cells per well in a final volume of $200 \mu \mathrm{l}$. Next, HeLa cells were allowed to adhere for next $16-18 \mathrm{~h}$. After $16 \mathrm{~h}$, cells were treated in triplicates as DMEM Control, BCEF: DMEM (1:1) and EFM: DMEM (1:1). Importantly, DMEM media control was prepared to truly represent the control with reference to EFM and BCEF. In this experiment, HeLa cells were incubated with above described treatment conditions for $48 \mathrm{~h}$ at $37^{\circ} \mathrm{C}$. At the end of experiment, medium from wells was aspirated. Further, cells were treated with $100 \mu \mathrm{l}$ of medium to avoid interference during absorbance. Then, 10 $\mu \mathrm{l}$ of (Thiazolyl blue tetrazolium) MTT solution was added to each respective well. Next, cells were then incubated for 2-4 $\mathrm{h}$ in dark. At the end of incubation and observation of purple color, $100 \mu \mathrm{l}$ of isopropanol was added to each well. The plate was gently stirred and pipetted in and out to allow the crystals to be dissolved. The cells were incubated for $2 \mathrm{~h}$ in dark and the absorbance was measured by using ELISA reader (ThermoFisher Scientific) at $570 \mathrm{~nm}$ and at a reference wavelength of $650 \mathrm{~nm}$ (Scudiero et al., 1988; Marks et al., 1992).

\section{Evaluation of apoptotic cell death by flow cytometry}

HeLa cells were seeded in duplicates into six well plate at the plating density of $15 \times 10^{4}$ cell per well. After 16-18 h of plating, HeLa cells were allowed to grow in the presence of DMEM control media, BCEF: DMEM $(1: 1)$ and EFM: DMEM $(1: 1)$ to observe the differential growth and survival. In this assay, a positive control of doxorubicin treatment to HeLa was done to observe apoptotic cells. Next, HeLa cells were incubated for $48 \mathrm{~h}$ under as the above mentioned routine growth condition. At the end of incubation, HeLa cells were collected by routine trypsinization and cell counting was performed using haemocytometer. Cell suspensions were centrifuged at 1,500 rpm for $3 \mathrm{~min}$. Finally, pellets were resuspended in $1 \mathrm{ml}$ of cold PBS buffer. Next, Cells were centrifuged and annexin binding buffer was added to the pellet. We performed this assay using Annexin V/FITC apoptosis detection kit (ThermoFisher) to stain HeLa cells for Annexin V and propidium iodide (PI). Tubes were kept for incubation at room temperature in dark for $15 \mathrm{~min}$ and Annexin binding buffer was added to all the tubes. Then, cells were analysed on BD FACSJazz Cytometer to count the live and dead cells. At least, 10,000 events were collected and analysed per measurement.

\section{Cell Cycle analysis of HeLa cells by flow cytometer}

The cell cycle analysis was done after the cells were treated for $48 \mathrm{~h}$ as mentioned earlier. After the treatment cells were harvested and washed with ice-cold $1 \mathrm{X}$ PBS for 3 times. After washing the cells were subjected to fixation by $70 \%$ ethanol with incubation at $4^{\circ} \mathrm{C}$ for $30 \mathrm{~min}$. The fixated cells were passed through cell strainer to eliminate the clumped cells. Further, the cells were washed with PBS and they were subjected to RNase $(5 \mu \mathrm{g} / \mathrm{ml})$ and PI $(10 \mu \mathrm{g} / \mathrm{ml})$. Finally, stained cells were analysed on BD FACSJazz using blue laser.

Asian Pacific Journal of Cancer Prevention, Vol 193309 


\section{Statistical analysis}

Each experiment was performed at least three times. Results are expressed as mean values \pm SD. Error bars represent mean $\pm \mathrm{SD}$ of the triplicates. Data from the different assays were statistically compared using paired Students t-test as Microsoft office excel 2013 statistical package. Statistical significance was acceptable to a level of $\mathrm{p}<0.05$. $* \mathrm{P}<0.05$ and $* * \mathrm{P}<0.005$ significantly different from DMEM media control.
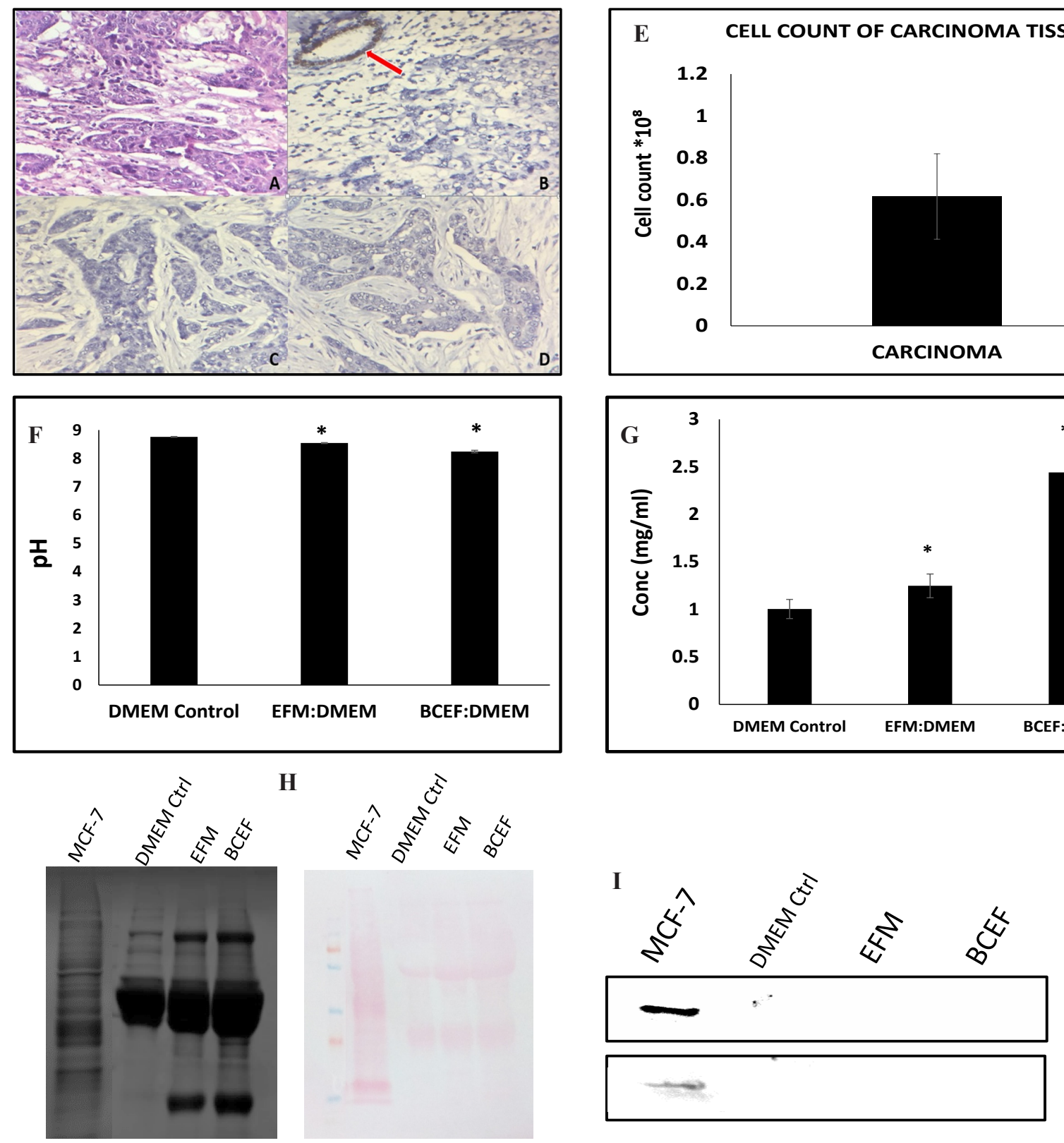

Figure 1. (Fig 1A-D) Represents the immunohistochemical images of ductal infiltrating breast carcinoma (Grade II). (Figure 1E) Represents the bar graph data of the total cell count from the tissue pellet obtained while processing the breast carcinoma tissue. The total cell count was recorded using Haemocytometer and data is presented number X10 ${ }^{8}$ total cell count.Error bars represent mean $\pm \mathrm{SD}$ of the triplicates. (Figure 1F) The graph represents $\mathrm{pH}$ of the samples DMEM Control, EFM: DMEM and BCEF: DMEM before the treatment to measure the hydrogen ion activity in the solution indicating the acidity or alkalinity. The diagram (Figure $1 \mathrm{G}$ ) is a representative bar graph data of protein concentration of the samples as DMEM Control, EFM: DMEM and BCEF: DMEM.Error bars represent mean \pm SD of the triplicates. (Figure 1H) Representative SDS-PAGE protein profiles of extracellular secreted components of breast carcinoma tissue and cells and Ponceau staining image of nitrocellulose membrane. (Figure 1I) represents image of immunoblot carried on three treatment samples with respect to intracellular protein $\mathrm{p} 53$ and cytochrome $\mathrm{b}$. 
triple negative status based on the receptor analysis using immunohistochemical method (Figure 1 B-D).

Next, we attempted to collect data to show that BCEF is free from lysed cellular content during its preparation. In that direction, we referred an earlier evidence, which showed that that approximate total number of cancer cells and cancer associated non-tumor cells can be in the range of $0.7-1 \times 10^{8}$ cells per $g$ of tumor tissue. Hence, it is pertinent to determine the number of cells in the pelleted fraction of breast cancer tissue sections used for the preparation of BCEF. This data will support the possibility of non-lysis of tumor cells during the preparation of BCEF. Therefore, total cell count of pelleted breast carcinoma tissue sample, which was stored after the collection of supernatant, which is also referred as BCEF was performed using standard haemocytometer based counting (Figure 1E). Data of cell count expressed as per g of breast cancer tissue is found to be in the range of $0.8-1 \times 10^{8}$ that corresponds to the earlier data reported with reference to approximate number of cells present in a tumor tissue. These data therefore support that the possibility of cellular lysis of tumor cell during preparation of BCEF is almost negligible. Here, one possible reason behind absence of lysis of tumor cell could be due to the use of DMEM complete media, which is an ideal isotonic medium added with serum that may protect the cell from lysis during homogenization. There are well known methodology that for cell lysis hypotonic solutions and detergents are recommended. Hence, this data clearly indicates that all the cells from the tissue definitely settled down in pelleted fraction and supernatant designated as BCEF contains predominantly extracellular factors of breast carcinoma tissue used in these experiments.

To exclude the possibility of changed $\mathrm{pH}$ of supernatant prepared from MCF-7 conditioned medium (EFM) and breast carcinoma tissue (BCEF) that might contribute toward possible effects upon the growth and survival of HeLa. To look into this aspect, we performed standard $\mathrm{pH}$ determination assay of DMEM control, EFM: DMEM $(1: 1)$ and BCEF: DMEM (1:1), as these different media compositions are used to assess the effects of breast carcinoma extracellular factors upon the HeLa growth and survival. As seen in the bar graph data (Figure 1F) $(\mathrm{p}=0.02), \mathrm{pH}$ of these media are found to be identical in the range of $\mathrm{pH}(8.1-8.3)$, which is normally required for the growth of cells in vitro. Therefore, data indicate that observed cytotoxicity to HeLa cells due to incubation with EFM: DMEM (1:1) and BCEF: DMEM (1:1) could not have occurred due to shift towards acidic $\mathrm{pH}$ environment. Instead of $\mathrm{pH}$ as a factor, data point toward factors available in EFM and BCEF that are responsible for the growth inhibition and toxicity of HeLa cells. Next, it was important to determine the extent of extracellular factors in the form of protein as a macromolecule in these EFM and BCEF components. Therefore, we estimated amount of protein in EFM: DMEM (1:1) and BCEF: DMEM (1:1) in comparison to DMEM medium control by Lowry method and the data is presented as mg per $\mathrm{ml}$ (Figure $1 \mathrm{G}$ ). The analysis of protein concentration suggests that amount of protein slightly increased in EFM: DMEM (1:1) and BCEF: DMEM (1:1) and this result is not unexpected as extracellular factors from breast carcinoma have been reported in the form of several proteins and peptides. At this point, this preliminary data support the possibility that these secreted factors in the form of proteins and peptides could be involved in the observed inhibitory effects in growth and proliferation of HeLa cells. In continuation, we also extended our efforts to perform SDS-PAGE protein profiling to know the nature of extracellular factors in EFM: DMEM (1:1) and BCEF: DMEM (1:1) (Figure 1H). SDS-PAGE profile indicates that several extra-protein bands of a wide range of molecular weight appeared to be accumulated in EFM and BCEF. In turn, analysis of SDS-PAGE profile data support the increased presence of protein amount in EFM and BCEF in comparison to DMEM control media, also the image of proteins transferred on nitrocellulose membrane stained with Ponceau is given. These SDS-PAGE and Ponceau stained data served as a normal control for the immunoblot data presented in this paper. Further, we also performed immunoblotting to confirm that EFM and BCEF are free from breast carcinoma cellular proteins. Hence, detection of representative cellular proteins p53 and cytochrome $\mathrm{b}$ are analysed and presented in (Figure 1I) and the data suggests that EFM: DMEM $(1: 1)$ and BCEF: DMEM (1:1) do not contain the cellular proteins from lysed breast carcinoma cells. Hence, this observation reinforces our view that EFM and BCEF as extracellular factors from breast cancer cells which are free from lysed cellular components either from in vitro culture of $\mathrm{MCF}-7$ or breast carcinoma tissue samples. Thus, observed increased protein content and its SDS-PAGE profile is potentially contributed from the extracellular factors released from MCF-7 into EFM and BCEF from breast carcinoma tissue.

\section{Evaluation of survival inhibition and cytotoxicity effects by EFM and BCEF}

In order to evaluate the survival inhibitory effects of EFM and BCEF, Trypan blue dye exclusion assay was performed on HeLa cells at the end of treatment. Representative photomicrographs are presented in Figure $2 \mathrm{~A}$, which qualitatively depicts the decrease in the total cell count for HeLa due to the treatment of breast cancer associated extracellular factors. For all these treated HeLa samples, total cell count and percent survival of cells were carried out employing haemocytometer and Trypan blue dye, respectively and data is given in Figure $2 \mathrm{~B}$ and $2 \mathrm{C}$. These quantitative data indicated that EFM can arrest HeLa cells growth to some extent, however, less than the effects of BCEF. Accordingly, effects of BCEF demonstrated clear reduction in total HeLa count up to $32 \%$ and also percent viability of HeLa cells decreased to $41 \%$ and $53 \%$ after treatment with EFM and $B C E F$ respectively, Figure $2 B(p=0.0058)$ and Figure $2 \mathrm{C}(\mathrm{p}=0.004)$.

To investigate the cytotoxic effects of BCEF and EFM upon HeLa cells, routinely used MTT assay was conducted and results are presented in the form of photomicrograph of formazan crystal formed due to enzymatic conversion by the metabolically active enzyme in Figure $2 \mathrm{D}$. The results are also shown in the form of absorbance based readings between different treatment conditions on 
Figure 2A

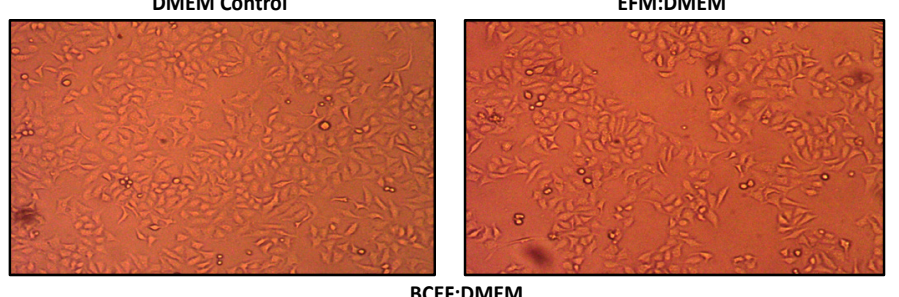

BCEF:DMEM
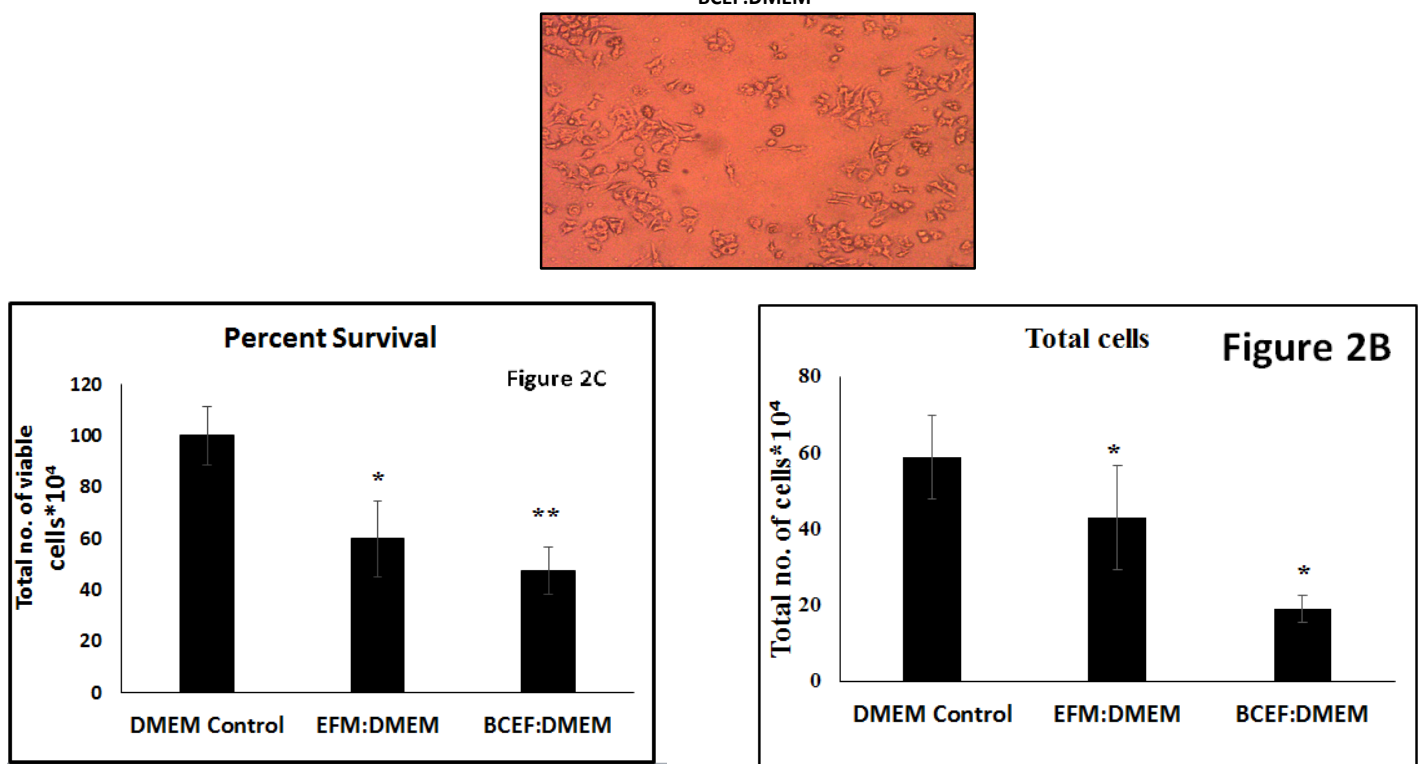

Figure 2D
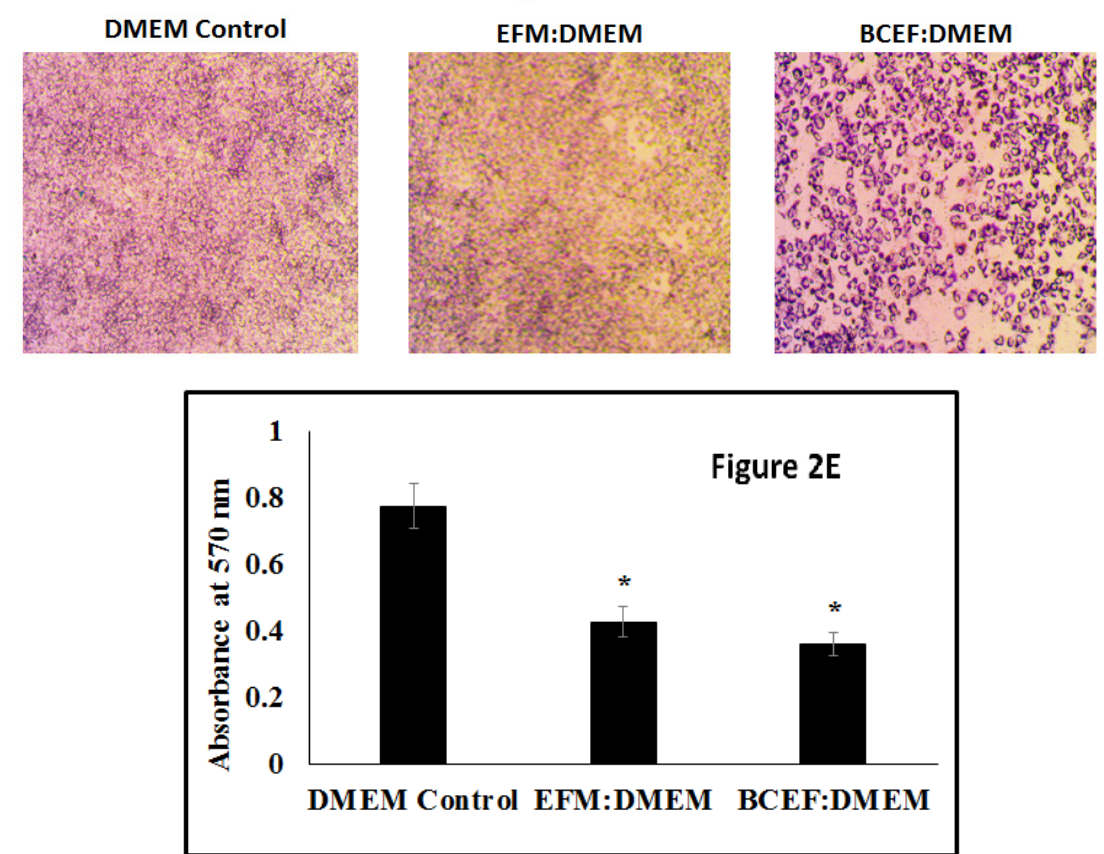

Figure 2. Haemocytometer and Microscopy based HeLa Cell Proliferation Data is Presented. (Figure 2A) represents the photomicrograph taken at 10X of HeLa growing in six well plate with different treatment condition whereas DMEM Control, EFM: DMEM and BCEF: DMEM. The Microscopy photographs were taken at the end of treatment at $48 \mathrm{~h}$. The Microscopy photographs were captured using light inverted microscope. (Figure 2B) is a graphical representative oftotal cells after treatment condition (DMEM Control DMEM Control, EFM: DMEM and BCEF: DMEM). The Total cells were recorded using Haemocytometer and data is presented as Percentage of Total Cell Count X $10^{4}$ in each treatment conditions. The Data are represented as Mean \pm SD. Each experiment was conducted independently four times. (Figure 2C) shows us the percent viability of cells after the respective treatment conditions. (Figure 2D) The photograph depicts the photomicrograph taken at 10X for HeLa cell growing in 96 Well Plate with different treatment condition (DMEM Control, EFM: DMEM and BCEF: DMEM). The microscopy photographs were taken at the end of MTT based cytotoxicity assay showing Formazan crystals formed, using 10 X Objective. (Figure 2E) Graphical representation of absorbance captured by ELISA plate reader with cells treated with DMEM Control DMEM Control, EFM: DMEM and BCEF: DMEM. Total cells were recorded by taking absorbance with ELISA reader. The Data are represented as Mean \pm SD. Each experiment was conducted independently three times. 
Figure 3A
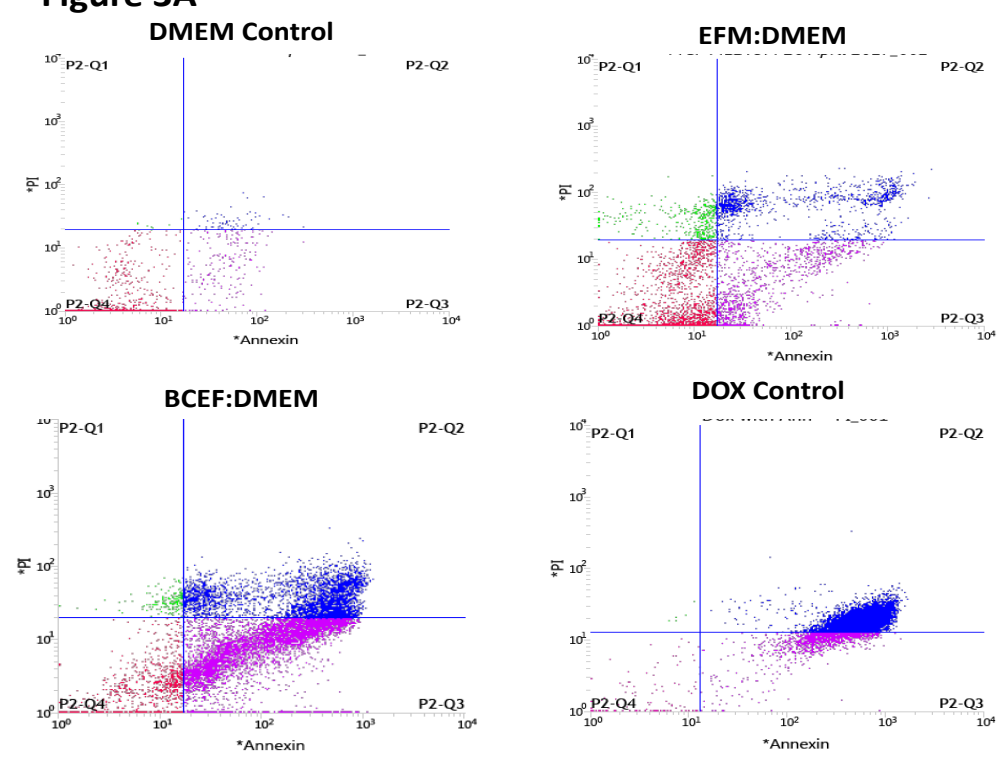

\section{Figure 3B}

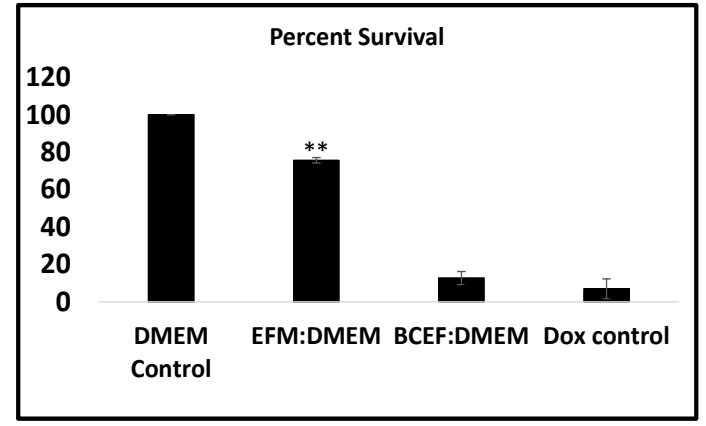

Figure $3 \mathrm{C}$

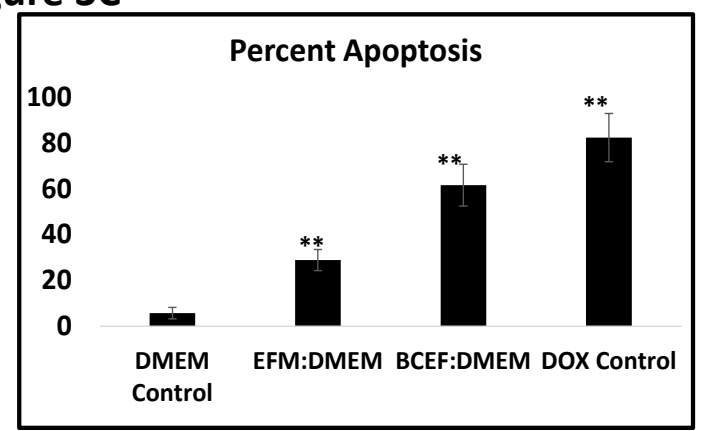

\section{Figure 3D}

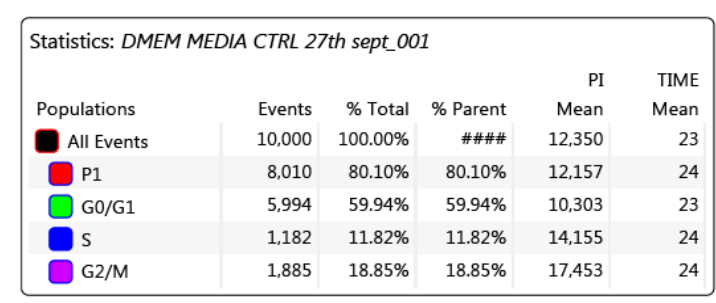

\begin{tabular}{|c|c|c|c|c|c|}
\hline \multicolumn{6}{|c|}{ Statistics: EFM 27th sept_001 } \\
\hline & & & & PI & TIME \\
\hline Populations & Events & $\%$ Total & \% Parent & Mean & Mean \\
\hline All Events & 10,000 & $100.00 \%$ & \#\#\# & 9,309 & 9 \\
\hline P1 & 7,911 & $79.11 \%$ & $79.11 \%$ & 8,578 & 9 \\
\hline $\mathrm{G} 0 / \mathrm{G} 1$ & 5,466 & $54.66 \%$ & $54.66 \%$ & 7,271 & 9 \\
\hline$s$ & 2,176 & $21.76 \%$ & $21.76 \%$ & 9,528 & 9 \\
\hline $\mathrm{G} 2 / \mathrm{M}$ & 2,363 & $23.63 \%$ & $23.63 \%$ & 12,127 & 9 \\
\hline
\end{tabular}

\begin{tabular}{|c|c|c|c|c|c|}
\hline \multicolumn{6}{|c|}{ Statistics: BCEF 27th sept_001 } \\
\hline & & & & PI & TIME \\
\hline Populations & Events & $\%$ Total & $\%$ Parent & Mean & Mean \\
\hline All Events & 10,000 & $100.00 \%$ & \#\#\# & 13,815 & 27 \\
\hline P1 & 5,856 & $58.56 \%$ & $58.56 \%$ & 15,085 & 27 \\
\hline G0/G1 & 2,447 & $24.47 \%$ & $41.79 \%$ & 11,043 & 26 \\
\hline $\mathrm{s}$ & 1,368 & $13.68 \%$ & $23.36 \%$ & 14,799 & 28 \\
\hline $\mathrm{G} 2 / \mathrm{M}$ & 1,801 & $18.01 \%$ & $30.75 \%$ & 18,932 & 29 \\
\hline
\end{tabular}

Figure 3E

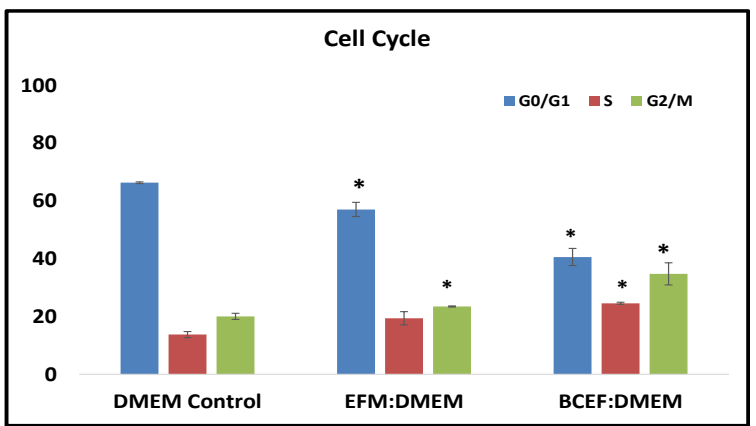

Figure 3. (Figure 3A) Data represents the cells stained with PI and Annexin V conjugated with FITC for analysis of apoptosis. The scatter plot of three treatment condition and cells treated with Doxorubicin as a positive control is shown. (Figure 3B and 3C) are representative graphs which depict percent survival and percent apoptosis derived from the statistical table of scatter plot respectively. (Figure 3D) depicting the cell cycle phases of cells after $48 \mathrm{~h}$ of treatment. (Fig 3E) Graphical form of the percentage distribution of cells in different cell cycle phases after $48 \mathrm{~h}$ of respective treatment.The apoptosis detection and cell cycle data was recorded using BDFACSJazz cytometer. Error bars represent mean $\pm \mathrm{SD}$ of the triplicates. 
HeLa cells (Figure 2E) $(p=0.003)$. The bar graph data of the absorbance was taken at $570 \mathrm{~nm}$. Data clearly suggest an appreciable extent of HeLa cell cytotoxicity due to both EFM and BCEF as compared to DMEM control medium. Here, MTT based data is also in accordance with the results of Trypan blue dye exclusion assay showing reduction in viable HeLa cells.

Flow cytometry analysis of HeLa proliferation and death

To evaluate further the effects of breast carcinoma non-cellular factors upon apoptotic death of HeLa cells, we estimated the presence of Annexin V/propidium iodide (PI) double positive HeLa cells in our data panel. As HeLa cells were allowed to grow in thepresence of EFM: DMEM (1:1) and BCEF: DMEM (1:1) as part of addition evidence to evaluate the effects of extracellular factors of breast carcinoma. These treatment conditions convincingly showed the presence of apoptosis in HeLa cells compared to the control DMEM media (Figure 3 A-C) $(p=0.003$ for Figure $3 B)(p=0.005$ for Figure $3 \mathrm{C})$. Results showed that in case of MCF-7 conditioned media, there is an increase in HeLa cell apoptotic death with respect to untreated control. Similarly, TNBC tissue non-cellular factors demonstrated more apoptotic death in HeLa cells, which is comparable to the results of doxorubicin treatment as the positive control (Kumar et al., 2017) which showed the presence of PI stained HeLa. The cell cycle analysis represented in Figure 3D and 3E depicts that the percentage of HeLa cells in $\mathrm{G} 0 / \mathrm{G} 1$ phase significantly decreased from $66 \%$ to $41 \%$ and at the same time $\mathrm{S}$ phase distribution of $\mathrm{HeLa}$ cells increased from $11 \%$ to $25 \%$.

\section{Discussion}

Many reports have been documented on drug resistance and its precision, but these often fail either due to resistance or lack of penetration of drug on the targeted site (Marusyk et al., 2012; Meacham and Morrison, 2013; Holohan et al., 2013; Ahuja et al., 2016). Tumor microenvironment comprises of cellular as well as non-cellular components and the difference in the microenvironment also termed as microenvironmental heterogeneity is the major cause behind cancer survival, progression, metastasis and drug resistance (Hanchen et al., 2007; Hanahan and Weinberg, 2011; Marusyk et al., 2012; Burrell et al., 2013; Meacham and Morrison, 2013; Chung et al. 2014; Alizadeh et al., 2015; Gkretsi et al., 2015; Yap et al., 2015; Sharma et al., 2016; Turner et al., 2017). To understand and address the uniqueness of one type of carcinoma over another, we have tested the potential of extracellular factors from one carcinoma to determine their ability to modulate growth and survival of another carcinoma. The term multiple cancer arises when there are two primary carcinomafound synchronously in an individual cancer patient. As the clinical reports suggest that the probability of breast and cervical carcinoma occurring together is very less (Jena et al., 2016; Verstovsek et al., 2000).

Our data emphasize a clear role of extracellular factors released from breast carcinoma tissue (TNBC types) and also from in vitro cultured MCF-7 cells on the growth and survival of HeLa cervical cancer cell. Essentially, this study is having another goal to address the issue of rare occurrence of multiple cancers (in this case cervical and breast carcinoma) and thatcan be explained by the possibility of antagonistic effects from one carcinoma towards another carcinoma. In this study, histopathological suggest the nature of breast cancer tissue with as TNBC typewitha moderately differentiated infiltrating ductal breast carcinoma. To understand the nature of BCEF and EFM, our data from cell counting, estimation of $\mathrm{pH}$ and western blot of cellular protein converge to a conclusion that possibility of leaking out of cellular content from carcinoma cells are minimal. Our data is also supported from an earlier report that probable total number of cells per gram of tumor tissue can be approximately in the range of 0.7-0.9X108, which well correlates with our cell counting data (Del Monte, 2009). In our western blot data, we did not find any presence of band for representative cellular proteins such as $\mathrm{p} 53$ and cytochrome $b$. Further, these data strengthens our claim that BCEF and ECF as supernatant are potentially free from intracellular content which could have contaminate during the BCEF preparation and that the BCEF was cell free. Therefore, sole contributions of extracellular secretion of breast cancer cells as EFM and BCEM are suggested. At the same time, other factors in the form of altered $\mathrm{pH}$, contamination from lysed cellular portions including lysosome released acid proteins and other classes of protease and DNAses are excluded in our case, which could potentially interfered with the observed growth inhibition and apoptotic death in HeLa. Authors would like support the possibility of absence of cellular lysis during the preparation of $\mathrm{BCEF}$, as extracting media is DMEM high glucose supplemented with serum. Since, we would like to refer to standard protocol having hypotonic nature and presence of detergents in the lysis buffer to obtain whole cell lysate or cellular fractionation from tumor tissue. In this case, the nature and composition of used homogenization medium suggest that it can act efficient isotonic and osmotic protectant to prevent the breakage of cell membrane during extraction. At the same time, authors monitored the obtained supernatant and cell pellets for the possible contamination from cellular lysis in the supernatant. In this pursuit, we have also tested the effects of BCEF and EFM upon the growth and survival of MDA-MB-231 and we did not find inhibitory role upon this TNBC cell line (Data not shown).

As it is already known that tumor microenvironment is rich in signals and soluble factors that create a favourable niche for a type of cancer to thrive and progress. However, possibilities of effects of these signals and soluble factors from one cancer types upon another cancer cell types may turn as synergistic or antagonistic. In this paper, anti-proliferative and cytotoxicity assays including Trypan blue dye exclusion and MTT assays suggest the compatible observations that EFM and BCEF can bring appreciable reduction the HeLa survival. However, these two assays are of preliminary nature, but suitability is highly appreciated in literature based on the sound reproducibility and unbiased observations (Scudiero et al., 1988; Strober et al., 2015). The outcome of these experiments suggest that extracellular factors secreted 
from breast carcinoma tissue and cells may have potential to interfere with the growth and survival of HeLa and this finding may propose that possible antagonistic cross-talk between breast carcinoma secreted factors and survival mechanism of HeLa.

Based on the clear observation of reduced proliferation and survival of HeLa due to incubation with extracellular factors from breast cancer cells, we asked the nature of retardation of growth and survival. As reported in earlier work, Annexin V/PI staining to HeLa cells can provide some clue about the nature and extent of cell death. Our data indicate thatthe presence of $50 \%$ increase in the apoptotic cells treated with BCEF in comparison to normal media control and this data is potentially compared with other apoptotic inducing drugs (Barthes et al., 2014). On the other side, the extracellular factors from MCF-7 also showed reduced cell viability and modest increase in apoptotic HeLa, however less profound in comparison to BCEF. Besides induction of apoptosis, our data clearly showed the cell cycle arrest of HeLa cells in the present of EFM and BCEF. These observations point that EFM and BCEF can bring cell cycle arrest at G2-M phase which is well supported by the other anti-cancer drug treatment approaches (Geoffrey et al., 1999; Singh et al., 2004; Sato et al., 2007). At the same time, we predict that certainly a set of soluble factors released from breast cancer cells may act as a signalling agents to activate apoptosis and G2-M cell arrest upon the target HeLa cells and able to show the anti-growth and proliferation potentials. These observations support the notion of intra- and inter tumor, and inter-cancer type's heterogeneity driven by non-cellular extracellular secreted factors.

Taken together, these results converge to the conclusion that extracellular factors collected from the TNBC tissue and MCF-7 cells could demonstrate a strong anti-growth and survival effects on the cervical cancer cell line HeLa. Further, these findings lend support to the well known phenomenon of cancer cachexia, which is described as survival tactics by cancerous cells to cause systemic release of some factors that prevent the development of malignancy at other sites of the body and disturb metabolisms of healthy tissues. A major explanation has been reported that supports the idea of cancer driven cachexia: by the autocrine and paracrine mediated actions of chemokines, cytokines, other soluble extra-cellular proteins/peptides secreted by a cancer tissue to constitute their own favourable niche and may serve in the form cachexins (Kirr et al., 2014; Alves et al., 2017; Zhang et al., 2017). These cachexins comprising of chemokines, cytokines and extra-cellular secreted proteins/peptides as heat shock proteins etc. have been suggested to play a crucial role in the cancer related cachexia. It may thus be appropriate to suggest that a set of chemokines/cytokines secreted by the tumor microenvironment may act as an anti-growth, anti-metabolic adaptation and anti-physiological survival. Therefore, it seems logical to propose that these anti-growth and metabolic starving strategies achieved through paracrine signaling may be responsible for the avoidance and suppressing the development of a distinct second primary malignancy in the same cancer patient. Hence, current insights suggest that non-cellular factors released from one carcinoma type may show inhibitory influence upon another genetically and epigenetically distinct carcinoma.

In conclusion, our data suggest that the extracellular factors collected from in vitro grown MCF-7 cells and breast carcinoma tissue which is confirmed as TNBC molecular types can potentially retard the growth of HeLa cells leading to apoptotic cell death. We speculate that these observations can be explained based on the fact that there are clear evidences of intra- and inter-tumor heterogeneity in tumour within one cancer type. Here, we have tested extracellular factors from one breast carcinoma type to other HeLa cervical carcinoma, which sounds logical as these two carcinoma types may have distinct tumour microenvironmental heterogeneity comprising of cellular and non-cellular extracellular factors. Possibly, defined non-cellular secreted components from one carcinoma type can show antagonistic role in other carcinoma type. Our results also support one possible reason behind rare occurrence of two or over two multiple cancers in the same individual like simultaneous report of breast carcinoma and cervical carcinoma.

\section{Declaration of interest}

Authors declare no conflict of interest.

\section{References}

Ahuja N, Sharma AR, Baylin SB (2016). Epigenetic therapeutics: A new weapon in the war against cancer. Annu Rev Med, 67, 73-89.

Alizadeh A, Aranda V, Bardelli A, et al (2015). Toward understanding and exploiting tumor heterogeneity. Nat Med, 21, 846-53.

Alves MJ, Figuerêdo RG, Azevedo FF, et al (2017). Adipose tissue fibrosis in human cancer cachexia: the role of TGF $\beta$ pathway. BMC Cancer, 17, 190.

Barthes J, Özçelik H, Hindié M, et al (2014). Cell microenvironment engineering and monitoring for tissue engineering and regenerative medicine: the recent advances. Biomed Res Int, 2014, 921905.

Burrell RA, McGranahan N, Bartek J, Swanton C (2013). The causes and consequences of genetic heterogeneity in cancer evolution. Nature, 501, 338-45.

Chung HW, Lim JB, (2014) Role of the tumor microenvironment in the pathogenesis of gastric carcinoma. World $J$ Gastroenterol, 20, 1667-80.

Del Monte U (2009). Does the cell number 109 still really fit one gram of tumor tissue?. Cell Cycle, 8, 505-6.

Geoffrey I Shapiro, J Wade Harper (1999). Anticancer drug targets: cell cycle and checkpoint control. J Clin Invest, 104, 1645-53.

Gkretsi V, Stylianou A, Papageorgis P, Polydorou C, Stylianopoulos T (2011). Remodeling components of the tumor microenvironment to enhance cancer therapy. Front Oncol, 5, 214.

Hanahan D, Weinberg RA (2011). Hallmarks of cancer: the next generation. Cell, 144, 646-74.

Hanchen Li, Xueli Fan, Houghton JM (2007). Tumor Microenvironment: The role of the tumor stroma in cancer. $J$ Cell Biochem, 101, 805-15.

Holohan C, Van Schaeybroeck S, Longley DB, Johnston PG, (2013). Cancer drug resistance: an evolving paradigm. Nat Rev Cancer, 13, 714-26.

Asian Pacific Journal of Cancer Prevention, Vol $19 \mathbf{3 3 1 5}$ 
Jena A, Patnayak R, Lakshmi AY, Manilal B, Reddy MK (2016). Multiple primary cancers: An enigma. South Asian J Cancer, 5, 29-32.

Kir S, White JP, Kleiner S, et al. (2014). Tumour-derived PTH-related protein triggers adipose tissue browning and cancer cachexia. Nature, 513, 100-4.

Kumar A, Bhatkar D, Jahagirdar D, Sharma NK (2017). Non-homologous end joining inhibitor SCR-7 to exacerbate low-dose doxorubicin cytotoxicity in HeLa cells. $J$ Cancer Prev, 22, 47-54.

Liu D, Chang C, Lu N, et al (2017). Comprehensive proteomics analysis reveals metabolic reprogramming of tumor-associated macrophages stimulated by the tumor microenvironment. J Proteome Res, 16, 288-97.

Louis KS, Siegel AC (2011). Cell viability analysis using trypan blue: manual and automated methods. Methods Mol Biol, 740, 7-12.

Marks DC, Belov L, Davey MW, Davey RA, Kidman AD (1992). The MTT cell viability assay for cytotoxicity testing in multidrug-resistant human leukemic cells. Leuk Res, 16, 1165-73.

Marusyk A, Almendro V, Polyak K (2012). Intra-tumour heterogeneity: a looking glass for cancer?. Nat Rev Cancer, 12, 323-4.

Mbeunkui F, Johann DJ Jr (2009). Cancer and the tumor microenvironment: a review of an essential relationship. Cancer Chemother Pharmacol, 63, 571-82

Meacham CE, Morrison SJ (2013). Tumour heterogeneity and cancer cell plasticity. Nature, 501, 328-37.

Sato M, Sagawa M, Nakazato T, Ikeda Y, Kizaki M (2017). A natural peptide, dolastatin 15 , induces $\mathrm{G} 2 / \mathrm{M}$ cell cycle arrest and apoptosis of human multiple myeloma cells. Int J Oncol, 30, 1453-9.

Scudiero DA, Shoemaker RH, Paull KD, et al (1998). Evaluation of a soluble tetrazolium/formazan assay for cell growth and drug sensitivity in culture using human and other tumor cell lines. Cancer Res, 48, 4827-33.

Sharma SH, Thulasingam S, Nagarajan S (2016). Chemopreventive agents targeting tumor microenvironment. Life Sci, 145, 74-84.

Singh SV, Herman-Antosiewicz A, Singh AV, Lew KL, Srivastava SK (2004). Sulforaphane-induced G2/M phase cell cycle arrest involves checkpoint kinase 2-mediated phosphorylation of cell division cycle 25C. J Biol Chem, 279, 25813-22.

Steinbichler TB, Dudás J, Riechelmann H, Skvortsova II (2017). The role of exosomes in cancer metastasis. Semin Cancer Biol, 44, 170-81.

Strober W (2015). Trypan blue exclusion test of cell viability. Curr Protoc Immunol, 111, A3.B.1-3.

Sung BH, Weaver AM (2017). Exosome secretion promotes chemotaxis of cancer cells. Cell Adh Migr, 11, 187-95.

Turner KM, Deshpande V, Beyter D, et al (2017). Extrachromosomal oncogene amplification drives tumour evolution and genetic heterogeneity. Nature, 543, 122-5.

Verstovsek S, Verschraegen CF, et al (2000). Synchronous primary cancers of the breast and cervix: planning multidisciplinary primary treatment. Am J Clin Oncol, 23, 99-103.

Weidle UH, Birzele F, Kollmorgen G, Rüger R (2017). The multiple roles of exosomes in metastasis.Cancer Genomics Proteomics, 14, 1-15.

Yap TA, Gerlinger M, Futreal PA, Pusztai L, Swanton C (2015). Intra-tumor heterogeneity: seeing the wood for the trees. Sci Transl Med, 4, 127ps10.

Yuan Y (2016). Spatial heterogeneity in the tumor microenvironment. Cold Spring Harb Perspect Med, 6, 8.
Zhang G, Liu Z1, Ding H, et al (2017). Tumor induces muscle wasting in mice through releasing extracellular Hsp70 and Hsp90. Nat Commun, 8, 589.

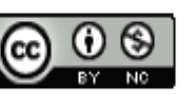

This work is licensed under a Creative Commons AttributionNon Commercial 4.0 International License. 